\title{
Fetuin-A and Fetuin-B in Non-Alcoholic Fatty Liver Disease: A Meta-Analysis and Meta-Regression
}

\author{
Xiongfeng Pan ${ }^{1}$, Atipatsa C. Kaminga ${ }^{1,2}$, Jihua Chen ${ }^{1} \mathbb{D}$, Miyang Luo ${ }^{3, *}$ and Jiayou Luo ${ }^{1, *}$ \\ 1 Xiangya School of Public Health, Central South University, Changsha 410008, China; pxfcsu@163.com (X.P.); \\ atipatsachiwanda@yahoo.co.uk (A.C.K.); chenjh@csu.edu.cn (J.C.) \\ 2 Department of Mathematics and Statistics, Mzuzu University, Mzuzu 999120, Malawi \\ 3 Saw Swee Hock School of Public Health, National University of Singapore, Singapore 999002, Singapore \\ * Correspondence: lmy4300@gmail.com (M.L.); jiayouluo@126.com (J.L.); \\ Tel.: +65-16-4988 (M.L.); +86-139-7484-1828 (J.L.); Fax: +86-731-8480-5454 (J.L.)
}

Received: 25 February 2020; Accepted: 9 April 2020; Published: 15 April 2020

check for updates

\begin{abstract}
The magnitude of the effect of fetuin-A and fetuin-B on non-alcoholic fatty liver disease (NAFLD) remains undefined. Therefore, the aim of this study was to synthesize previous findings to obtain a reliable estimation of this relationship. This study was registered in PROSPERO with the number CRD42019126314. Studies published not later than March 2019, examining the relationship between fetuin-A, fetuin-B, and NAFLD, were identified by a systematic search in the electronic databases of the Web of Science, PubMed, Embase, and Cochrane Library. Pooled estimates of standardized mean difference (SMD), calculated using the random-effects model in a meta-analysis, were applied to estimate the strength of the association between fetuin-A, fetuin-B, and NAFLD. Thirty publications were identified and analyzed based on specified inclusion criteria. Collectively, they consisted of 3800 NAFLD participants and 3614 controls. Compared with the controls, significant higher values of the fetuin-A (SMD $=0.83,95 \% \mathrm{CI}$ : 0.59 to $1.07, \mathrm{Z}=6.82, p<0.001$ ) and fetuin-B $(\mathrm{SMD}=0.18,95 \% \mathrm{CI}: 0.02$ to $0.33, \mathrm{Z}=2.27, p=0.023$ ) were observed in NAFLD patients. Meanwhile, in the subgroup analysis, the effect value of fetuin-A in the NASH group was significantly higher than that in the NAFL group ( $p=0.036$ ). The findings of this study suggest that elevated fetuin-A and fetuin-B may independently indicate the occurrence of NAFLD. Nevertheless, further research is needed to confirm these results.
\end{abstract}

Keywords: fetuin-A; fetuin-B; non-alcoholic fatty liver disease; meta-analysis; meta-regression

\section{Background}

Non-alcoholic fatty liver disease (NAFLD) is now highly prevalent and often related to complications such as liver failure, cirrhosis, and liver cancer. Thus, it has become a major health concern and new worldwide epidemic. Basically, NAFLD is a chronic hepatic steatosis from non-alcoholic fatty liver (NAFL) to non-alcoholic steatohepatitis (NASH) [1]. However, its exact pathogenesis is still obscure. Notwithstanding, there has been increasing evidence to indicate that hepatokines such as fetuin-A and fetuin-B may influence the prognoses of NAFLD and associated complications [2]. Besides, epidemiological investigations also showed that fetuin-A and fetuin-B not only played a role in the development of obesity, hyperglycemia, and hypertriglyceridemia, but also led to chronic diseases, such as metabolic syndrome (MetS), type 2 diabetes mellitus (T2DM), and cardiovascular disease (CVD) $[3,4]$. In addition, current evidence in genetics suggests that, compared with the healthy controls, patients with liver steatosis have higher expression of fetuin-A mRNA in hepatocytes and serum fetuin-A concentrations [5]. The different amounts of this protein in the two groups could be due to the fact that fetuin-A (a2HS-glycoprotein), which is a glycoprotein produced by the adipose 
tissue and liver, has a dual function, that is, it restrains the insulin receptor and brings about insulin resistance in the pancreas, skeletal muscle, and hepatocytes [6]. It also has an inverse relationship with tumor necrosis factor (TNF- $\alpha$ ), interleukin-6 (IL-6), and C-reactive protein (CRP) level, which is the critical factor in systemic inflammation in NAFLD [7]. However, unlike fetuin-A, fetuin-B is secreted by hepatocytes of a high-fat diet and does not induce pro-inflammatory signals and macrophage activation. Rather, it increases in steatosis and induces insulin resistance. In addition, experimental studies have shown that serum levels of fetuin- $B$ are not associated with adiponectin levels. Although some previous studies indicated that both fetuin- $A$ and fetuin-B belonged to the proteins attributed to the cystatin superfamily with shares of $22 \%$ sequence similarity, other studies revealed the differential effects of fetuin-A and fetuin-B as regards to the pathophysiology of fatty liver [8]. For example, the concentrations of fetuin-A and fetuin-B have been shown to be positively correlated and this relationship played a role in NAFLD, even after controlling for gender and age [8].

To sum up, there is clear evidence that fetuin-A and fetuin-B are complex metabolic phase reactants that have controversial roles in NAFLD. Therefore, the aim of this study was to synthesize previous findings to obtain a reliable estimation of the relationship between fetuin-A, fetuin-B, and NAFLD.

\section{Methods}

\subsection{Search Strategy}

This study was carried out using the guidelines provided in the Cochrane handbook (version 5.1.0) for systematic reviews and meta-analysis, whereas the results were reported according to the Preferred Reporting Items for Systematic Reviews and Meta-Analyses (PRISMA) [9]. In addition, this study was registered with the International Prospective Register of Systematic Reviews PROSPERO (CRD42019126314) by XP.

Studies published between January 1959 and March 2019, examining the relationship between fetuin-A, fetuin-B, and NAFLD, were identified by a systematic search in the electronic databases of the Web of Science, PubMed, Embase, and Cochrane Library. Detailed search strategies were designed with help from experienced librarians and are shown in the appendix of the Supporting Information. Details of the search strategy are presented in Supplementary File. For example, in the PubMed database, the literature search was carried out using the following search strategy:

((alpha 2 HS Glycoprotein[Title/Abstract] OR alpha(2) HS Glycoprotein[Title/Abstract] OR alpha-2HS Glycoprotein[Title/Abstract] OR alpha 2HS Glycoprotein[Title/Abstract] OR alpha2HS Glycoprotein[Title/Abstract] OR Fetuin-A[Title/Abstract] OR Fetuin-A[Title/Abstract] OR AHSG Protein[Title/Abstract] OR alpha2 Heremans-Schmid Glycoprotein[Title/Abstract] OR alpha-2-HS-Glycoprotein[Title/Abstract] OR fetuins[Title/Abstract] OR Fetuin[Title/Abstract] OR Fetuin-B[Title/Abstract] OR Fetuin-B[Title/Abstract])) AND (Non-alcoholic Fatty Liver Disease OR NAFLD OR Nonalcoholic Fatty Liver Disease OR Fatty Liver, Non-alcoholic OR Fatty Livers, Non-alcoholic OR Liver, Nonalcoholic Fatty OR Livers, Nonalcoholic Fatty OR Nonalcoholic Fatty Liver OR Nonalcoholic Fatty Livers OR Nonalcoholic Steatohepatitis OR Nonalcoholic Steatohepatitides OR Steatohepatitides, Nonalcoholic OR Steatohepatitis, Nonalcoholic OR Non-alcoholic Fatty Liver Disease OR non-alcoholic steatohepatitis OR NASH OR Fatty Liver OR non-alcoholic steatohepatitis OR non-alcoholic fatty liver disease).

\subsection{Eligibility Criteria}

Titles, abstracts, and full texts of the studies yielded after searching in the electronic databases were screened to meet the following inclusion criteria: (1) Studies were case-control studies; (2) studies reported a criteria used to diagnose NAFLD; (3) studies provided mean and standard deviation (SD) of fetuin-A and fetuin-B, or these could be obtained from respective authors on request; (4) studies were peer-reviewed before publication; and (5) studies were published in English. On the contrary, the exclusion criteria were: (1) Studies were case reports or reviews; (2) studies reported NAFLD in 
combination with other diseases; (3) studies had fetuin-A and fetuin-B pharmacologically challenged before their measurements; (4) studies did not examine humans; and (5) studies were grey literature (unpublished literature). Two researchers [KA and AK] independently identified the eligible studies, and their discrepancies were resolved by involving the third researcher [JL].

\subsection{Data Extraction}

Data related to the following variables were extracted: (1) The first author's name and year of publication; (2) subject's characteristics such as Body Mass Index (BMI), mean age (mean, SD), and gender; (3) region of study; (4) participant's laboratory characteristics such as aspartate aminotransferase (AST), alanine aminotransferase (ALT), glutamyl transpeptidase (GGT), low-density lipoprotein (LDL), high-density lipoprotein (HDL), triglycerides (TG), and insulin resistance index (HOMA-IR); (5) sample characteristics such as the material of sample, and mean and SD of fetuin-A and fetuin-B; and (6) fetuin-A and fetuin-B sample test methods, and storage temperatures. Two researchers [KA and AK] conducted data extraction independently using EpiData 3.0 and Microsoft Excel 2007. Any discrepancies between them were resolved by consensus.

\subsection{Quality Evaluation}

The non-randomized Newcastle-Ottawa Scale (NOS) was used to assess the quality of this study [10]. It is a 9-star rating system designed for non-randomized studies, which includes 3 domains and 8 items. The 3 domains consisted of the following broad perspectives: (1) Selection; (2) comparability; and (3) outcome. According to the NOS, study quality was classified as low, moderate, and high according to the scores, $0-3,4-6$, and 7-9, respectively.

\subsection{Statistical Analysis}

The random effects model was used to pool the estimates under discussion because of the varying populations and criteria used to define outcomes across the eligible studies. This model, therefore, was used to merge standardized mean differences (SMDs) and corresponding 95\% confidence intervals (CIs), to compare the concentrations of fetuin-A and fetuin-B between NAFLD patients and the controls, which was calculated as Cohen's d [11]. The pooled effect value, SMD, was considered to be high if greater than 0.8 , moderate if between 0.5 and 0.8 , or low if lower than 0.5 . In addition, heterogeneity between-study was assessed using the Cochrane $Q$ statistic, and the level of heterogeneity was measured by the $\mathrm{I}^{2}$ statistic [12]. Similarly, the heterogeneity was considered low if the $\mathrm{I}^{2}$ statistic was lower than $25 \%$, moderate if it was $25 \%-75 \%$, and high when it was greater than $75 \%$. Additionally, the overall results were divided into the subgroups, including NASH patients and the control group, and NAFL patients and the control group. In addition, subgroup analysis was conducted to explore whether samples, storage methods, or ethnicity had any influence on the results. However, the subgroup analysis of fetuin-B was not carried out due to inadequate information related to fetuin-B. Furthermore, meta-regression analyses were conducted to explore the source of heterogeneity when this was significantly high. Subjects' characteristics such as BMI and mean age (mean, SD); and participant's laboratory characteristics such as AST, ALT, GGT, LDL, HDL, and TG were taken into account when exploring the source of heterogeneity. Additionally, a sensitivity analysis was performed by repeating the meta-analysis when each was omitted in turn. Publication bias was assessed by examining the symmetry of funnel plots when the number of studies reporting the primary outcomes was at least 10, and the significance of the symmetry was tested using the Egger's linear regression test [13]. All analyses were done with the 'meta' and 'metafor' packages in R software (version R 3.4.3, MathSoft, Englewood, NJ, USA). In addition, all the statistical tests were two-sided, and $p$-values less than 0.05 indicated significant results for all statistical tests. 


\section{Results}

\subsection{Literature Search}

Figure 1 shows an overview of the search results and screening process. A total of 831 articles were found in the four electronic databases as follows: 181 from Embase, 17 from the Cochrane Library, 266 from Web of Science, and 367 from PubMed. After excluding the duplicates, 728 articles were retained. The titles and abstracts of the 728 articles were reviewed, and this resulted in 519 articles excluded because they did not meet the inclusion criteria. Then, a full-text review of 209 articles further excluded 179 studies, of which 108 were unrelated to the topic of this study, 19 had no data on fetuin-A and fetuin-B, 35 did not report means and SDs of fetuin-A and fetuin-B concentrations, 8 did not compare fetuin-A and fetuin-B concentrations between NAFLD patients and a control group, 3 were reviews, and 6 did not report the results of a control group. Finally, a total of 30 articles met the inclusion criteria and were included in the final meta-analysis.
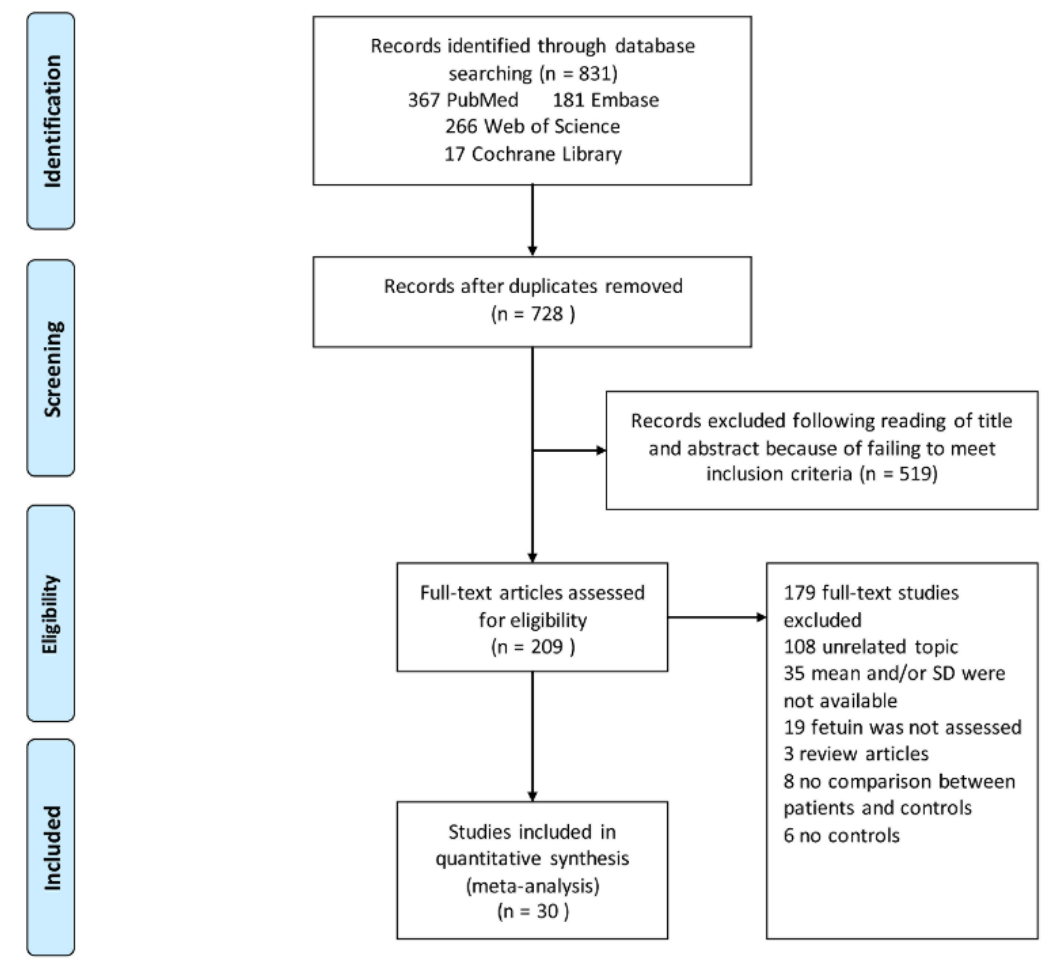

Figure 1. Flowchart of study selection. Showing the process by which retrieved studies from the databases were assessed and selected or excluded. Preferred reporting items for systematic reviews and meta-analyses (PRISMA) diagram for study search.

\subsection{Characteristics of Eligible Studies}

Characteristics of the 30 eligible studies are presented in Table 1, which shows the patients' characteristics such as age, gender, BMI, and region; sample characteristics such as the source of the sample, and mean and SD of fetuin-A and fetuin-B concentrations. Characteristics of clinical parameters of the studies included for the meta-analysis of fetuin-A, fetuin-B, and NAFLD are presented in Table 2. Participant's laboratory characteristics such as AST, ALT, GGT, LDL, HDL, HOMA-IR, and TG; fetuin-A and fetuin-B sample assay methods; and storage temperatures. A total of 28 analyses of fetuin-A (Figure 2a) and 5 analyses of fetuin-B (Figure 2b) were identified from the 30 eligible studies. Altogether, these studies compared fetuin-A concentrations between 2109 NAFLD patients and 2457 controls; and fetuin-B concentrations between 1691 NAFLD patients and 1157 controls. The NOS scores of these studies varied between 5 and 8 , with 21 studies graded as high quality and 9 as moderate quality. 
Table 1. Characteristics of the studies included for the meta-analysis of fetuin-A, fetuin-B, and NAFLD.

\begin{tabular}{|c|c|c|c|c|c|c|c|c|c|c|c|c|}
\hline Study & & Material & Country & NOS & Male Gender, $\boldsymbol{n}(\%)$ & BMI & Mean Age & Study Type & Diagnosis of NAFLD & $\begin{array}{c}\text { Overnight } \\
\text { Fasting Blood }\end{array}$ & Methods & Frozen \\
\hline (Ballestri et al., 2013) & [14] & Serum & Italy & 7 & $20(69.0)$ & $29.2 \pm 5.0$ & $64.5 \pm 10.5$ & Case-control & Ultrasonography & Yes & ELISA & NR \\
\hline (Celebi et al., 2015) & [7] & Plasma & Turkey & 8 & $69(100.0)$ & $28.57 \pm 3.40$ & $31.8 \pm 5.6$ & Case-control & $\begin{array}{l}\text { Liver biopsy } \\
\text { specimens scored }\end{array}$ & Yes & ELISA & $-80^{\circ} \mathrm{C}$ \\
\hline (Cui et al., 2017) & [15] & Serum & China & 6 & $58(73.4)$ & $26.0 \pm 3.0$ & $42.8 \pm 10.8$ & Case-control & $\begin{array}{l}\text { Ultrasonography } \\
\text { Ung }\end{array}$ & Yes & ELISA & NR \\
\hline (Dogru et al., 2013) & [16] & Plasma & Turkey & 7 & $31(100.0)$ & $28.4 \pm 2.97$ & $31.0 \pm 3.9$ & Case-control & $\begin{array}{c}\text { Liver biopsy } \\
\text { specimens scored }\end{array}$ & Yes & ELISA & $-80^{\circ} \mathrm{C}$ \\
\hline $\begin{array}{l}\text { (Ebert et al., 2017) } \\
\text { (Şiraz Ü et al., 2017) }\end{array}$ & $\begin{array}{l}{[17]} \\
{[18]}\end{array}$ & $\begin{array}{l}\text { Serum } \\
\text { Serum }\end{array}$ & $\begin{array}{l}\text { Germany } \\
\text { Turkey }\end{array}$ & $\begin{array}{l}6 \\
7\end{array}$ & $\begin{array}{c}52(51.4) \\
4(50.0)\end{array}$ & $\begin{array}{l}28.7 \pm 6.8 \\
20.4 \pm 5.6\end{array}$ & $\begin{array}{l}62.3 \pm 16.4 \\
14.0 \pm 3.7\end{array}$ & $\begin{array}{l}\text { Case-control } \\
\text { Case-control }\end{array}$ & $\begin{array}{l}\text { Ultrasonography } \\
\text { Ultrasongography }\end{array}$ & $\begin{array}{l}\text { Yes } \\
\text { Yes }\end{array}$ & $\begin{array}{l}\text { ELISA } \\
\text { ELISA }\end{array}$ & $\begin{array}{c}\mathrm{NR} \\
-80^{\circ} \mathrm{C}\end{array}$ \\
\hline (Haukeland et al., 2010) & {$[19]$} & Plasma & Norway & 8 & $77(62.0)$ & $23.9 \pm 3.0$ & $62.7 \pm 7.5$ & Case-control & $\begin{array}{l}\text { Liver biopsy } \\
\text { specimens scored }\end{array}$ & No & ELISA & $-80^{\circ} \mathrm{C}$ \\
\hline (Haukeland et al., 2012) & [20] & Plasma & Norway & 8 & $67(60.0)$ & $30.5 \pm 4.3$ & $46.5 \pm 11.6$ & Case-control & $\begin{array}{l}\text { Liver biopsy } \\
\text { specimens scored }\end{array}$ & No & ELISA & $-80^{\circ} \mathrm{C}$ \\
\hline (El-Ashmawy and Ahmed, 2019) & [21] & Serum & Egypt & 8 & $55(52.4)$ & $27.8 \pm 4.1$ & $53.4 \pm 9.2$ & Case-control & $\begin{array}{l}\text { Specimens sorea } \\
\text { Ultrasonography }\end{array}$ & Yes & ELISA & NR \\
\hline (Kahraman et al., 2013) & [5] & Serum & Turkey & 6 & $55(23.1)$ & $37.6 \pm 1.2$ & $41.9 \pm 0.9$ & Case-control & $\begin{array}{c}\text { Liver biopsy } \\
\text { specimens scored }\end{array}$ & No & ELISA & $4^{\circ} \mathrm{C}$ \\
\hline $\begin{array}{l}\text { (Kurtoglu et al., 2015) } \\
\text { (Lebensztejn et al, 2014) }\end{array}$ & {$[22]$} & $\begin{array}{l}\text { Serum } \\
\text { Serum }\end{array}$ & $\begin{array}{l}\text { Turkey } \\
\text { Poland }\end{array}$ & $\begin{array}{l}5 \\
7\end{array}$ & $\begin{array}{l}4(50.0) \\
12(63.3)\end{array}$ & $\begin{array}{l}25.1 \pm 3.0 \\
27.4 \pm 7.3\end{array}$ & $\begin{array}{l}15.3 \pm 3.7 \\
13.0 \pm 3.9\end{array}$ & $\begin{array}{l}\text { Case-control } \\
\text { Case-control }\end{array}$ & $\begin{array}{l}\text { Ultrasonography } \\
\text { Ultrasonography }\end{array}$ & $\begin{array}{l}\text { No } \\
\text { No }\end{array}$ & $\begin{array}{l}\text { ELISA } \\
\text { ELISA }\end{array}$ & $\begin{array}{l}\text { NR } \\
\text { NR }\end{array}$ \\
\hline (von Loeffelholz et al., 2016) & [24] & Plasma & Germany & 8 & $35(46)$ & $27.3 \pm 1.1$ & $61.0 \pm 2.0$ & Case-control & $\begin{array}{c}\text { Liver biopsy } \\
\text { specimens scored }\end{array}$ & Yes & ELISA & $-80^{\circ} \mathrm{C}$ \\
\hline $\begin{array}{l}\text { (Li et al., 2018) } \\
\text { (Lin et al. 2018) }\end{array}$ & {$[25]$} & $\begin{array}{l}\text { Serum } \\
\text { Serum }\end{array}$ & $\begin{array}{l}\text { China } \\
\text { China }\end{array}$ & $\begin{array}{l}7 \\
5\end{array}$ & $\begin{array}{l}283(36.2) \\
398(65.3)\end{array}$ & $\begin{array}{l}28.2 \pm 3.1 \\
25.1 \pm 3.0\end{array}$ & $\begin{array}{l}53.9 \pm 7.0 \\
61.9 \pm 7.3\end{array}$ & $\begin{array}{l}\text { Case-control } \\
\text { Case-control }\end{array}$ & $\begin{array}{l}\text { Ultrasonography } \\
\text { Ultrasonography }\end{array}$ & $\begin{array}{l}\text { Yes } \\
\text { No }\end{array}$ & $\begin{array}{l}\text { ELISA } \\
\text { ELISA }\end{array}$ & $\begin{array}{l}\mathrm{NR} \\
\mathrm{NR}\end{array}$ \\
\hline (Mondal et al., 2018) & [27] & Serum & India & 8 & $\begin{array}{l}26(56.5) \\
26.5)\end{array}$ & $\begin{array}{l}27.5 \pm 6.0 \\
27.5\end{array}$ & $\begin{array}{l}49.6 \pm 12.2 \\
49.5\end{array}$ & Case-control & $\begin{array}{l}\text { Ultrasonography } \\
\text { Ulits }\end{array}$ & $\begin{array}{l}\text { Yes } \\
\text { Yes }\end{array}$ & ELISA & $-80^{\circ} \mathrm{C}$ \\
\hline (Nascimbeni et al., 2018) & [2] & Serum & Italy & 7 & $34(76)$ & $27.3 \pm 19.1$ & $69.0 \pm 11.7$ & Case-control & Ultrasonography & Yes & ELISA & $\mathrm{NR}$ \\
\hline (Nascimbeni et al, 2015) & {$[28]$} & Serum & Italy & 7 & $62(78.2)$ & $27.0 \pm 4.0$ & $71.0 \pm 10.0$ & Case-control & Ultrasonography & Yes & ELISA & NR \\
\hline (Ou et al, 2012a) & [29] & Serum & China & 7 & $51(56.7)$ & $26.1 \pm 3.1$ & $62.0 \pm 10.0$ & Case-control & Ultrasonography & Yes & ELISA & $\mathrm{NR}$ \\
\hline (Ou et al., 2012b) & [30] & Serum & China & 7 & $25(55.6)$ & $25.8 \pm 3.0$ & $61.0 \pm 10.0$ & Case-control & Ultrasonography & Yes & ELISA & $\mathrm{NR}$ \\
\hline $\begin{array}{l}\text { (Pampanini et al., 2018) }\end{array}$ & [31] & Serum & Sweden & 6 & $5(55.6)$ & $31.0 \pm 9.0$ & $8.9 \pm 2.3$ & Case-control & Ultrasonography & Yes & ELISA & $\mathrm{NR}$ \\
\hline (Rametta et al, 2014) & [32] & Plasma & Italy & 8 & $106(77.3)$ & $26.9 \pm 3.4$ & $49.7 \pm 12.1$ & Case-control & $\begin{array}{c}\text { Liver biopsy } \\
\text { specimens scored }\end{array}$ & Yes & ELISA & $-80^{\circ} \mathrm{C}$ \\
\hline (Reinehr and Roth, 2008) & [33] & Serum & USA & 8 & $9(75.0)$ & $27.6 \pm 3.8$ & $10.6 \pm 2.8$ & Case-control & $\begin{array}{l}\text { Specimens scored } \\
\text { Ultrasonography }\end{array}$ & Yes & ELISA & $-80^{\circ} \mathrm{C}$ \\
\hline (Roshdy et al, 2015) & [34] & Serum & Egypt & 5 & $\begin{array}{l}24(77.4) \\
\end{array}$ & $41.26 \pm 11.2$ & $27.0 \pm 2.7$ & Case-control & Ultrasonography & No & ELISA & NR \\
\hline (Sato et al., 2015) & {$[35]$} & Serum & Japan & 6 & $151(86.3)$ & $26.5 \pm 3.6$ & $56.4 \pm 6.9$ & Case-control & $\begin{array}{l}\text { Ultrasonography } \\
\text { Liver biopsy }\end{array}$ & Yes & ELISA & $-80^{\circ} \mathrm{C}$ \\
\hline (Sonmez et al., 2015) & [36] & Serum & Turkey & 8 & $17(70.0)$ & $27.72 \pm 1.99$ & $32.0 \pm 6.0$ & Case-control & $\begin{array}{l}\text { Liver biopsy } \\
\text { specimens scored }\end{array}$ & Yes & ELISA & $-80^{\circ} \mathrm{C}$ \\
\hline (Tanoglu et al., 2015) & [37] & Serum & Turkey & 5 & $35(83.3)$ & $29.72 \pm 3.91$ & $37.0 \pm 6.0$ & Case-control & $\begin{array}{l}\text { Liver biopsy } \\
\text { specimens scored }\end{array}$ & No & ELISA & NR \\
\hline (Wong et al., 2015) & [38] & Serum & China & 7 & $143(54.4)$ & $25.3 \pm 3.4$ & $51.0 \pm 9.0$ & Case-control & Ultrasonography & Yes & ELISA & NR \\
\hline $\begin{array}{l}\text { (Yilmaz et al., 2010) } \\
\text { (Zhu et al. 2017) }\end{array}$ & {$[39]$} & $\begin{array}{l}\text { Serum } \\
\text { Serum }\end{array}$ & $\begin{array}{l}\text { Turkey } \\
\text { China }\end{array}$ & $\begin{array}{l}8 \\
7\end{array}$ & $\begin{array}{l}46(46.5) \\
54(58.7)\end{array}$ & $\begin{array}{l}30.7 \pm 4.9 \\
258+2.5\end{array}$ & $\begin{array}{r}47.0+9.0 \\
58.4+10.9\end{array}$ & $\begin{array}{l}\text { Case-control } \\
\text { Case-control }\end{array}$ & $\begin{array}{l}\text { Ultrasonography } \\
\text { Ultrasonography }\end{array}$ & Yes & $\begin{array}{l}\text { ELISA } \\
\text { ELISA }\end{array}$ & $-80^{\circ} \mathrm{C}$ \\
\hline
\end{tabular}

NOS, Newcastle-Ottawa Scale; BMI, Body Mass Index; ELISA, enzyme-linked immunosorbent assay; NR, not report; NAFLD, non-alcoholic fatty liver disease. 
Table 2. Characteristics of clinical parameters of the studies included for the meta-analysis of fetuin-A, fetuin-B, and NAFLD.

\begin{tabular}{|c|c|c|c|c|c|c|c|c|}
\hline Study & & AST (IU/mL) & $\operatorname{ALT}(\mathrm{IU} / \mathrm{mL})$ & GGT (IU/mL) & $\mathrm{TG}(\mathrm{mmol} / \mathrm{L})$ & LDL (mmol/L) & HDL (mmol/L) & HOMA-IR \\
\hline (Ballestri et al., 2013) & [14] & 22.00 & 27.00 & 23.00 & 1.86 & 1.16 & 0.40 & 1.50 \\
\hline (Celebi et al., 2015) & [7] & 52.00 & 116.00 & 60.00 & 2.00 & 1.21 & 0.41 & 3.50 \\
\hline (Cui et al., 2017) & [15] & 21.00 & 21.00 & 24.00 & 1.17 & 2.46 & 1.39 & 3.27 \\
\hline (Dogru et al., 2013) & [16] & 48.00 & 103.00 & 59.00 & 1.66 & 1.28 & 0.40 & 3.35 \\
\hline (Ebert et al., 2017) & [17] & 80.00 & 88.00 & 97.00 & 1.60 & 3.10 & 1.30 & NR \\
\hline (Şiraz Ü et al., 2017) & [18] & 17.00 & 17.00 & 11.00 & 1.05 & 0.87 & 0.61 & NR \\
\hline (Haukeland et al., 2010) & [19] & 83.00 & 81.00 & 55.00 & 1.73 & 0.39 & 0.11 & 2.11 \\
\hline (Haukeland et al., 2012) & [20] & 87.00 & 89.00 & 57.00 & 1.80 & 0.35 & 0.12 & 2.21 \\
\hline (El-Ashmawy and Ahmed, 2019) & [21] & 35.00 & 45.00 & 53.00 & 1.91 & 1.08 & 0.45 & 4.90 \\
\hline (Kahraman et al., 2013) & [5] & 28.00 & 35.00 & 39.00 & NR & NR & NR & NR \\
\hline (Kurtoglu et al., 2015) & [22] & 19.00 & 21.00 & 13.00 & NR & NR & NR & NR \\
\hline (Lebensztejn et al., 2014) & [23] & 55.00 & 57.00 & 30.00 & 1.80 & 1.08 & 0.42 & 3.67 \\
\hline (von Loeffelholz et al., 2016) & [24] & 45.00 & 80.00 & 90.00 & 1.25 & 1.19 & 0.45 & 3.30 \\
\hline (Li et al., 2018) & [25] & 83.00 & 79.00 & 51.00 & 2.17 & 3.69 & 1.31 & 4.09 \\
\hline (Lin et al., 2018) & [26] & 53.00 & 56.00 & 33.00 & NR & NR & NR & 3.23 \\
\hline (Mondal et al., 2018) & [27] & 61.00 & 60.00 & 26.00 & 1.80 & 3.20 & 1.00 & 1.10 \\
\hline (Nascimbeni et al., 2018) & [2] & 21.00 & 23.00 & 30.00 & 1.18 & 1.10 & 0.40 & 1.80 \\
\hline (Nascimbeni et al., 2015) & [28] & 27.00 & 25.00 & 36.00 & 1.21 & 1.13 & 0.55 & 1.91 \\
\hline (Ou et al., 2012a) & [29] & 28.00 & 31.00 & 36.00 & 1.51 & 1.30 & 0.48 & 1.13 \\
\hline (Ou et al., 2012b) & [30] & 31.00 & 29.00 & 33.00 & 1.44 & 1.33 & 0.53 & 2.90 \\
\hline (Pampanini et al., 2018) & [31] & 29.00 & 31.00 & 30.00 & NR & NR & NR & NR \\
\hline (Rametta et al., 2014) & [32] & 65.00 & 60.00 & 89.00 & 1.35 & 1.21 & 0.47 & 2.50 \\
\hline (Reinehr and Roth, 2008) & [33] & 28.00 & 28.00 & 47.00 & 1.12 & 2.98 & 1.35 & 3.90 \\
\hline (Roshdy et al., 2015) & [34] & 25.00 & 31.00 & 37.00 & NR & NR & NR & NR \\
\hline (Sato et al., 2015) & [35] & 46.00 & 33.00 & 66.00 & 1.56 & 1.31 & 0.54 & NR \\
\hline (Sonmez et al., 2015) & [36] & 61.00 & 126.00 & 67.00 & 1.91 & 2.93 & 1.04 & 3.92 \\
\hline (Tanoglu et al., 2015) & [37] & 37.00 & 35.00 & 88.00 & NR & NR & NR & NR \\
\hline (Wong et al., 2015) & [38] & 22.00 & 32.00 & 35.00 & 1.60 & 3.20 & 1.30 & 2.50 \\
\hline (Yilmaz et al., 2010) & [39] & 42.00 & 68.00 & 47.00 & 1.80 & 1.42 & 0.46 & 3.80 \\
\hline (Zhu et al., 2017) & [40] & 22.00 & 38.00 & 30.00 & 1.51 & 3.20 & 1.20 & NR \\
\hline
\end{tabular}

AST, aspartate aminotransferase; ALT, alanine aminotransferase; GGT, glutamyl transpeptidase; LDL, low-density lipoprotein; HDL, high-density lipoprotein; TG, triglycerides; and HOMA-IR, insulin resistance index. 


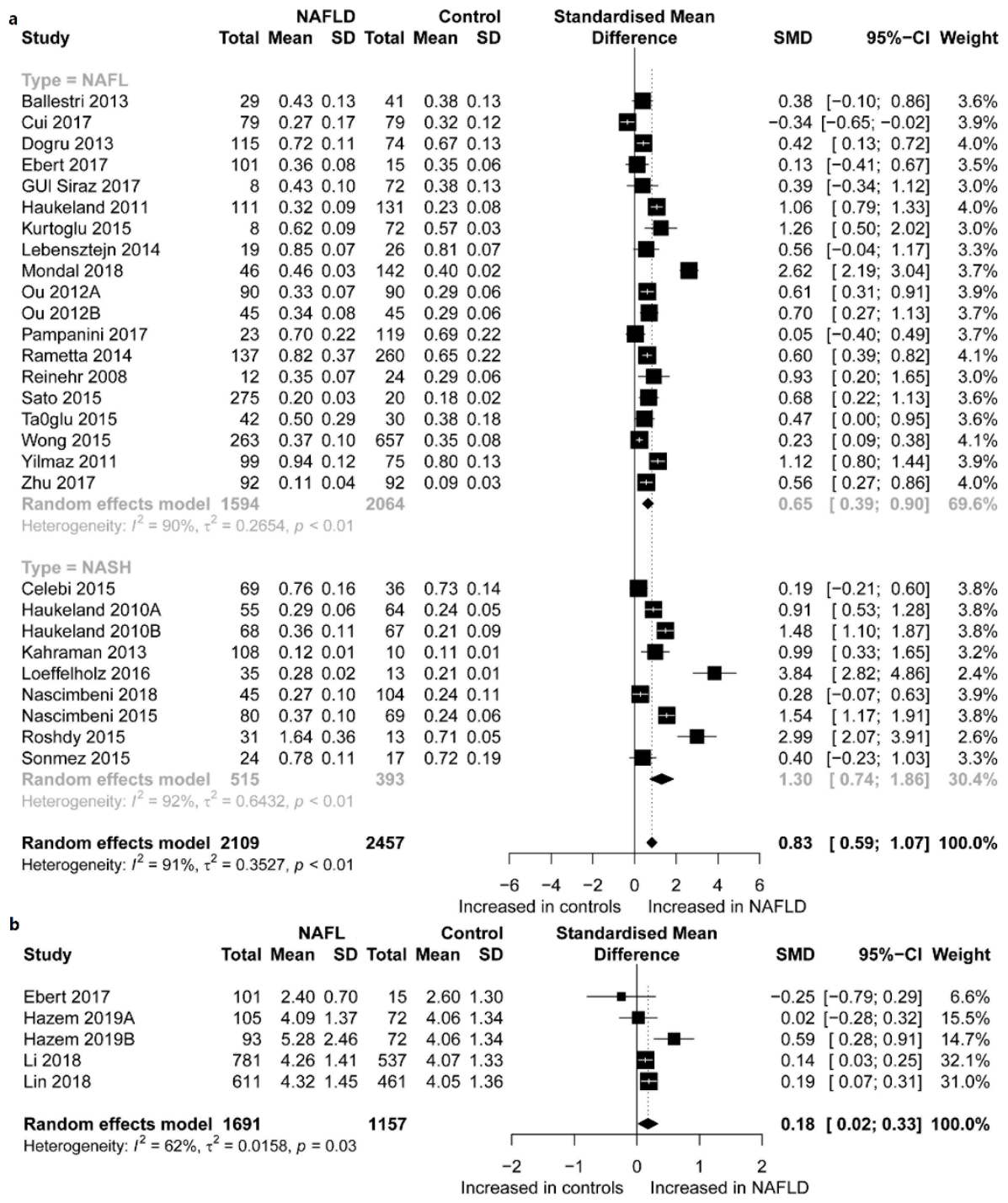

Figure 2. Forest plots of the standardized mean differences (SMDs) of fetuin-A concentrations (a), and fetuin-B concentrations (b) between the NAFLD participants and controls. The SMDs represent the effect sizes of fetuin-A, and fetuin-B concentrations on NAFLD. Each data marker represents a study, and the size of the data marker is proportional to the total number of individuals in that study. The summary effect sizes of fetuin- $A$, and fetuin-B concentrations are denoted by a diamond sign. The abbreviations in the forest plots are translated as follows: NAFLD, non-alcoholic fatty liver disease; NASH, non-alcoholic steatohepatitis; NAFL, non-alcoholic fatty liver; SMD, standardized mean difference.

\subsection{Overall Comparison}

Figure 2 presents forest plots of the standardized mean differences (SMDs) of fetuin-A and fetuin-B concentrations between the NAFLD participants and controls. Overall, taking the studies which compared fetuin-A concentrations between patients with NAFLD and the controls, it was found that fetuin-A concentrations were significantly higher in NAFLD patients than in the controls, with high effect (SMD $=0.83,95 \%$ CI: 0.59 to $1.07, p<0.001)$, and heterogeneity was considerable $\left(\mathrm{I}^{2}=91.1 \%\right)$. In addition, subgroup analysis found that fetuin-A concentrations were significantly higher in the NAFL patients than in the controls, with high effect (SMD $=0.65,95 \%$ CI: 0.39 to $0.90, p<0.001$ ); and in the NASH patients than in the controls, with high effect (SMD $=1.30,95 \%$ CI: 0.74 to 1.86, $p<0.001$ ), but both with high heterogeneity $\left(\mathrm{I}^{2}=90.0 \%\right.$ and $91.8 \%$, respectively). Moreover, the effect value, 
SMD, was significantly higher in the NASH group than in the NAFL group $(p=0.036)$. However, subgroup analysis showed no difference in the effect values of fetuin-A for plasma and serum samples $(p=0.282)$, and with respect to RACE $(p=0.155)$. Nonetheless, the effect values of fetuin-A in relation to storage methods were significantly different, and the effect values of fetuin-A according to frozen storage temperature were higher $(p=0.045)$ (Table 3). On the other hand, taking the studies which compared fetuin-B concentrations between patients with NAFLD and the controls, it was found that fetuin-B concentrations were higher in patients with NAFLD than in the controls, with low effect (SMD $=0.18,95 \%$ CI: 0.02 to $0.33, p=0.023)$, and moderate heterogeneity $\left(\mathrm{I}^{2}=62.3 \%\right)$.

Table 3. Subgroup analyses of fetuin-A between NAFLD participants and controls.

\begin{tabular}{|c|c|c|c|c|c|c|c|}
\hline & \multirow{2}{*}{$\begin{array}{c}\text { Number of } \\
\text { Studies }\end{array}$} & \multirow{2}{*}{ SMD $(95 \% \mathrm{CI})$} & \multirow{2}{*}{$\mathrm{Z}$} & \multirow{2}{*}{$p$-Value } & \multicolumn{3}{|c|}{ Heterogeneity } \\
\hline & & & & & Q Statistic (DF; $p$-Value) & $\tau^{2}$ & $\mathrm{I}^{2}$ \\
\hline \multicolumn{8}{|c|}{ Material } \\
\hline Plasma & 7 & $1.0426[0.6067 ; 1.4786]$ & 4.69 & 0.000 & $68.216<0.0001$ & 0.30 & $91.20 \%$ \\
\hline Serum & 21 & $0.7546[0.4626 ; 1.0466]$ & 5.07 & 0.000 & $224.3520<0.0001$ & 0.40 & $91.10 \%$ \\
\hline \multicolumn{8}{|c|}{ Study region } \\
\hline Asia race & 15 & $0.6741[0.3594 ; 0.9888]$ & 4.20 & 0.000 & $160.4214<0.0001$ & 0.33 & $91.30 \%$ \\
\hline $\begin{array}{c}\text { Caucasian race } \\
\text { Frozen }\end{array}$ & 13 & $1.0238[0.6580 ; 1.3897]$ & 5.49 & 0.000 & $119.2012<0.0001$ & 0.38 & $89.90 \%$ \\
\hline Yes & 14 & $0.9508[0.5704 ; 1.3312]$ & 4.9 & 0.000 & $163.5313<0.0001$ & 0.46 & $92.10 \%$ \\
\hline No & 14 & $0.5940[0.3070 ; 0.8809]$ & 4.06 & 0.000 & $108.1013<0.0001$ & 0.24 & $88.00 \%$ \\
\hline
\end{tabular}

Subgroup analyses are performed to compare the concentration of each fetuin-A between NAFLD and the controls. Heterogeneity was quantified using $\mathrm{I}^{2}$ and its significance was tested using the $\mathrm{Q}$ statistics. SMD, standardized mean difference; DF, degrees of Freedom; NAFLD, non-alcoholic fatty liver disease.

Additionally, the results of meta-regression analysis (with respect to Age, AST, BMI, ALT, GGT, TG, LDL, and HDL) of fetuin-A and fetuin-B are shown in Figures 3 and 4, respectively. As regards to fetuin-A and NAFLD, the following clinical characteristics had a positive effect on the SMD: BMI $(\beta \mathrm{BMI}=0.0636, \mathrm{SE}=0.0437, p=0.158)$, age $(\beta$ age $=0.0057, \mathrm{SE}=0.0090, p=0.529), \mathrm{AST}(\beta \mathrm{AST}=$ $0.0052, \mathrm{SE}=0.0077, p=0.502), \mathrm{ALT}(\beta \mathrm{ALT}=0.0018, \mathrm{SE}=0.0054, p=0.734), \mathrm{GGT}(\beta \mathrm{GGT}=0.0031, \mathrm{SE}=$ $0.0074, p=0.674)$, and TG ( $\beta$ TG $=0.0321, \mathrm{SE}=0.6628, p=0.962)$. However, subsequent meta-regression analysis revealed a negative effect of $\mathrm{LDL}(\beta \mathrm{LDL}=-0.0976, \mathrm{SE}=0.1854, p=0.604)$ and HDL $(\beta \mathrm{HDL}$ $=-0.4701, \mathrm{SE}=0.4258, p=0.282$ ) on SMD. Figure 3 shows each data point overlapping into a circle as illustrated by meta-regression models with $95 \%$ CIs. The size of a circle represents the weight of the associated data point such that the larger the circle the greater the impact.

Nonetheless, as regards fetuin-B and NAFLD, the following clinical characteristics had a negative effect on SMD: BMI ( $\beta$ BMI $=-0.0374, \mathrm{SE}=0.1057, p=0.747)$, age $(\beta$ age $=-0.0236, \mathrm{SE}=0.0316, p=$ $0.509), \operatorname{ALT}(\beta \mathrm{ALT}=-0.0080, \mathrm{SE}=0.0069, p=0.331), \mathrm{AST}(\beta \mathrm{AST}=-0.0056, \mathrm{SE}=0.0058, p=0.406)$, GGT $(\beta$ GGT $=-0.007, \mathrm{SE}=0.0007, p=0.406), \mathrm{LDL}(\beta \mathrm{LDL}=-0.1100, \mathrm{SE}=0.1519, p=0.544)$ and HDL $(\beta \mathrm{HDL}=-0.3586, \mathrm{SE}=0.4076, p=0.472)$. However, subsequent meta-regression analysis revealed a positive effect of TG on SMD ( $\beta$ TG $=0.5838$, SE $=0.9718, p=0.609$ ).

Sensitivity analysis showed that there was a small change in SMD and the corresponding $95 \%$ CI when each study was removed in turn, indicating that the current overall meta-analysis data were relatively stable. Publication bias was not reported for fetuin-B since the number of fetuin-B studies was less than 10. However, there was lack of symmetry in the shape of the Egger's funnel plot for fetuin-A, and the Egger's test was significant $(t=2.32$, $p$-value $=0.028)$, implying that there may be publication bias for this comparison. 

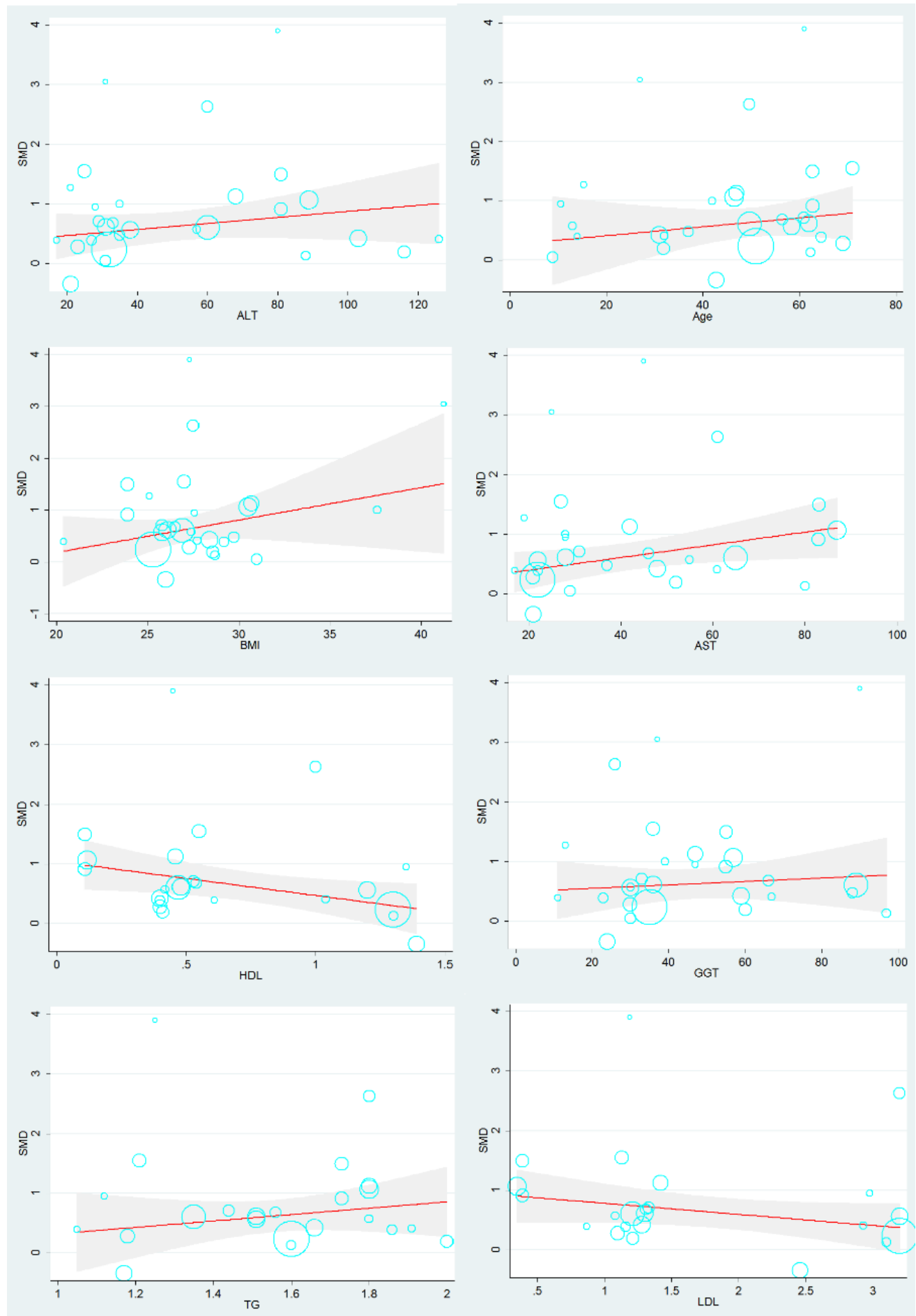

Figure 3. Dose-response relationships between clinical parameters of fetuin-A and SMD value outcomes based on data from each study. Each data point overlaps into a circle. The size of a circle represents the weight of the corresponding data point, and the larger the circle, the greater the impact. NAFLD, non-alcoholic fatty liver disease; AST, aspartate aminotransferase; ALT, alanine aminotransferase; GGT, glutamyl transpeptidase; LDL, low-density lipoprotein; HDL, high-density lipoprotein; TG, triglycerides; BMI, Body Mass Index; SMD, standardized mean difference. 

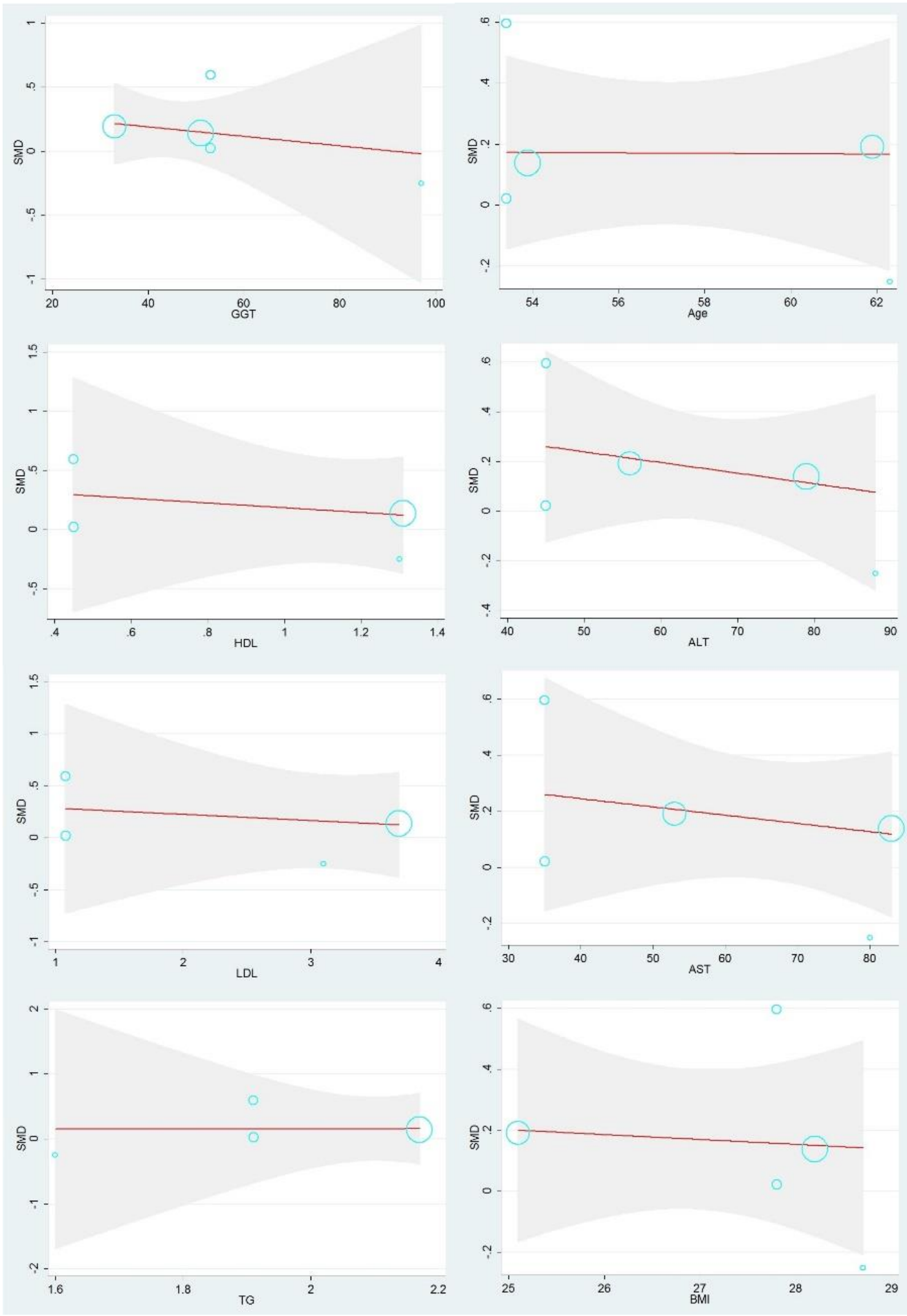

Figure 4. Dose-response relationships between clinical parameters of fetuin-B and SMD value outcomes based on data from each study. Each data point overlaps into a circle. The size of a circle represents the weight of the corresponding data point, and the larger the circle, the greater the impact. NAFLD, non-alcoholic fatty liver disease; AST, aspartate aminotransferase; ALT, alanine aminotransferase; GGT, glutamyl transpeptidase; LDL, low-density lipoprotein; HDL, high-density lipoprotein; TG, triglycerides; BMI, Body Mass Index; SMD, standardized mean difference. 


\section{Discussion}

This study has confirmed that there may be a relationship between fetuin-A, fetuin-B, and NAFLD. Specifically, there were higher concentrations of fetuin-A and fetuin-B in NAFLD patients than in the controls. The effect size of elevated fetuin-A concentrations on NAFLD was strong (SMD =0.83), thus supporting the previous hypothesis that there are raised levels of fetuin-A in NAFLD. In addition, subgroup analysis showed that the effect value of fetuin-A concentrations was significantly higher on the NASH patients than on the NAFL patients, which may indicate that fetuin-A played an important role in the pathophysiological process of NAFL to NASH. This inference could not be made on fetuin-B due to a lack of relevant studies on the relationship between fetuin-B concentrations and NASH. Therefore, the changes of fetuin-B in NASH should be studied accordingly in the future. However, subgroup analysis showed no difference in the effect values of fetuin-A for plasma and serum samples, and with respect to RACE, whereas the effect values of fetuin-A in relation to storage methods were significantly different, and the effect values of fetuin-A according to frozen storage temperature were higher. Thus, these findings suggest that the effects of fetuin-A were stable in relation to sample sources, storage methods, and race. However, future research should try to use cold chain to transport and store fetuin-A. Noteworthy, similar results were found between fetuin-A concentrations and other diseases. For example, fetuin-A levels were increased in other glucose and lipid metabolism disorders such as type 2 diabetes mellitus and metabolic syndrome. Although NAFLD and these other diseases are similarly identified as chronic diseases, the role of fetuin-A in NAFLD is likely to be more complex.

Notwithstanding, a recent study showed some hypothetical mechanisms about the relationship between fetuin-A and NAFLD. Specifically, that study suggested that a high-fat diet would bring excessive free fatty acids and cause hepatocytes and adipocytes to secrete fetuin-A, which can send chemical attraction signals and induce macrophages to infiltrate into adipose tissue [41]. In this regard, active macrophages increase the secretion of inflammatory cytokines, such as TNF- $\alpha$ and IL- 6 , which can further aggravate hepatocyte steatosis. Furthermore, existing data suggested that free fatty acids can increase the activity of nuclear factor $\kappa \mathrm{B}(\mathrm{NF}-\kappa \mathrm{B})$ by inhibiting Adenosine 5-monophosphate-activated protein kinase (AMPK); and can also enhance the activity of NF-kB by activating ERK 1/2 [42]. Given that NF- $\mathrm{B}$ plays an important role in up-regulating the expression of the fetuin-A gene and further increasing the level of fetuin-A mRNA, it brings about the overloaded fetuin-A in the cells, which may directly stimulate sterol regulatory element-binding protein-1c (SREBP-1C). It is generally acknowledged that the up-regulation of lipogenic enzymes by SREBP-1C is conducive to the accumulation of triacylglycerol [43]. An illustration of this series of hypotheses is shown in Figure 5 (by AK).

In addition, it has been confirmed from extensive studies of genetic epidemiology that polymorphisms in the gene encoding fetuin-A, the alpha-2-Heremans-Schmid glycoprotein (AHSG) gene, may affect glucose and lipid metabolism disorders' pathophysiology progression in some higher-risk groups. Furthermore, metabolic syndrome and type 2 diabetes mellitus are concerned with the single nucleotide polymorphisms (SNPs) of two risk alleles in AHSG (rs2518136 and rs4917) [44]. Therefore, these results suggest that AHSG and SNPs are recognized as a susceptibility locus for glucose and lipid metabolism disorders, which can further induce NAFLD progression.

In addition, clinical and experimental studies concurred with the hypothesis of a relationship between NAFLD and fetuin-A. Besides, it is well known that fetuin-A is significantly increased in hepatocellular injury and mediates insulin resistance as well as impairs glucose tolerance [45]. Moreover, accumulating clinical evidence has shown that increased fetuin-A concentrations in centripetal obesity individuals may lead to the development of NAFLD in this group [45]. 


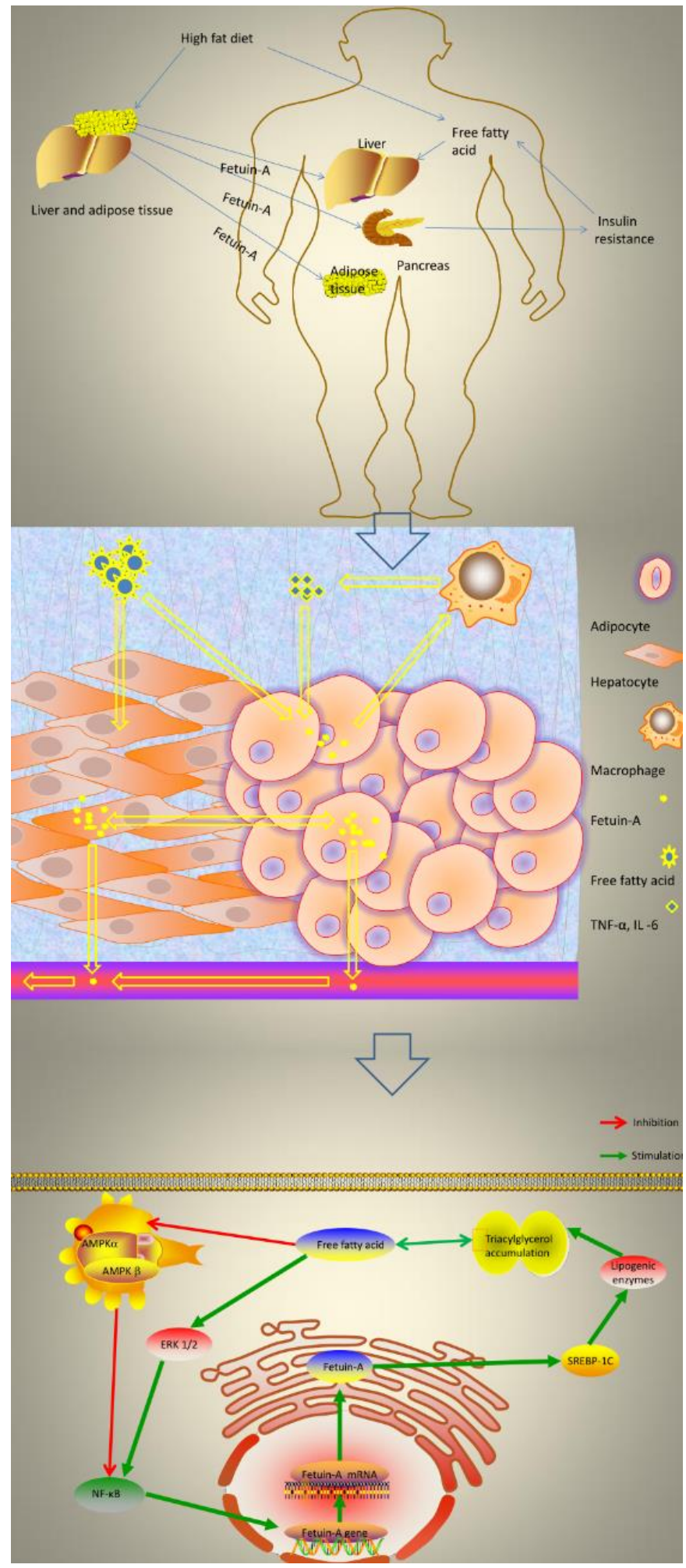

Figure 5. Summary of the hypothetical mechanism of fetuin-A in NAFLD. Figure 5 Schematic representation of the effects of fetuin-A on glucolipid metabolism signaling cascade in NAFLD that explain positive effects of these factors on glucolipid control. NAFLD, non-alcoholic fatty liver disease; IL -6: Interleukin-6; TNF- $\alpha$ : Tumor Necrosis Factor; AMPK: AMP-activated protein-kinase; NF-кB, nuclear factor $\mathrm{\kappa B}$; ERK 1/2, Extracellular signal-regulated kinases 1 and 2; SREBP- 1C, sterol regulatory element-binding protein-1c. 
Although previous studies have shown that fetuin-A and fetuin-B may be affected by other clinical parameters in NAFLD, this study has shown that fetuin-A might be a potential predictor of the occurrence of NAFLD, independent of age, AST, BMI, ALT, GGT, TG, LDL, and HDL. However, HDL and LDL had no significant influence on the effect size of fetuin-A in NAFLD.

Considering that increased activity of GGT, AST, and ALT generally indicates chronic and progressive hepatitis lesions, it is no wonder that GGT, AST, and ALT showed a positive influence on NAFLD [46]. As regards TG, Verras and colleagues reported that serum fetuin-A levels were significantly positively correlated with serum TG, even after controlling for sex and age, and this is in agreement with the findings of this study [47]. As for BMI and age, a recent study of genetic epidemiology indicated that BMI was significantly related to fetuin-A gene AHSG rs2248690 in an Asian population. In addition, Marhaug and colleagues found that fetuin-A levels were significantly higher in adult populations than in children and adolescents [48].

Despite the reasons for the negative effects of the levels of LDL and HDL found in this study, not being well known, it is common for LDL and HDL cholesterols to play a counterproductive role and a protective role, respectively, in NAFLD and other metabolic diseases. Thus, it is likely that LDL levels might be affected by fetuin-A. This phenomenon is relevant to the impairment of SIRT1 and AMPK energy sensors, in which fetuin-A may play an intermediary role in lipid-induced hepatic fat lesions [49]. Further, this may be connected to the reduction of LDL secreted directly into the blood after hepatic fat lesions [50]. However, these assumptions are based on cross-sectional analyses and do not imply established causality. Therefore, it is of great importance to perform prospective studies to evaluate the effect of fetuin-A on these indicators in patients with NAFLD at different stages.

However, unlike for fetuin- $A$, there was a negative linear relationship between fetuin-B and age, AST, BMI, ALT, GGT, HDL, and LDL, but a positive linear relationship between fetuin-B and TG. Even though these results seem beyond our understanding, previous studies have shown that there was a great positive linear relationship between the natural progression of fetuin-B in NAFLD and hepatic steatosis. Therefore, this may explain why the relationship between these indicators and fetuin-B was different from that between these indicators and fetuin-A. Although there may be a negative correlation between fetuin-B and liver fibrosis, this study did not consider exploring sources of heterogeneity with respect to liver fibrosis because the liver fibrosis scores of the patients varied considerably across the eligible studies. Thus, the relationship between fetuin-B and liver fibrosis needs to be further studied [17]. In addition, due to the small number of studies included in the meta-regression analysis, the statistical capacity may be limited. Therefore, these results need to be understood with caution.

This meta-analysis has some limitations. First, these findings were obtained from case-control studies, which cannot make causal inferences. Therefore, prospective studies should be considered in the future to explore the relationship between fetuin-A, fetuin-B, and NAFLD at different stages. Second, confounding factors that may affect fetuin-A and fetuin-B concentrations, such as race and cigarette smoking, were not analyzed in this study because these were not reported in the eligible studies. Third, studies on fetuin-A and fetuin-B concentrations between NAFLD patients and the controls showed high heterogeneity, which is not surprising given the large differences with respect to the study area and study population. Therefore, future original studies should consider making these investigations on homogeneous samples.

\section{Conclusions}

To sum up, the findings of this study showed that there were significantly higher fetuin-A and fetuin-B concentrations in NAFLD patients than in the controls. Thus, fetuin-A and fetuin-B might be potential predictors of the occurrence of NAFLD. Notably, fetuin-A may play an important role in the pathophysiological process of NAFL to NASH. These findings, therefore, provide a rationale for evaluating fetuin-A and fetuin-B in the pathophysiological process of NAFLD, and might open up new perspectives in early diagnosis, identification of novel biomarkers, and providing novel targets for pharmacological interventions. 
Supplementary Materials: The following are available online at http://www.mdpi.com/1660-4601/17/8/2735/s1, Supplementary File: Search strategies. Details of the search strategy.

Author Contributions: Conceptualization and design, X.P., A.C.K., and J.L.; data collection and analysis, J.C. and J.L.; writing—original draft preparation, M.L., A.C.K., and J.L.; writing—review and editing, M.L., A.C.K., and J.L. All authors have read and agreed to the published version of the manuscript.

Funding: This work was supported by grants from the Chinese Key Project of the National Natural Science Foundation (81872641 to JL).

Acknowledgments: We are grateful to Central South University Library for the assistance during the literature search.

Conflicts of Interest: The authors declare no conflict of interest.

\section{References}

1. Sanyal, A.; Charles, E.D.; Neuschwander-Tetri, B.A.; Loomba, R.; Harrison, S.A.; Abdelmalek, M.F.; Lawitz, E.J.; Halegoua-DeMarzio, D.; Kundu, S.; Noviello, S.; et al. Pegbelfermin (BMS-986036), a PEGylated fibroblast growth factor 21 analogue, in patients with non-alcoholic steatohepatitis: A randomised, double-blind, placebo-controlled, phase 2a trial. Lancet (Lond. Engl.) 2019, 392, 2705-2717. [CrossRef]

2. Nascimbeni, F.; Romagnoli, D.; Ballestri, S.; Baldelli, E.; Lugari, S.; Sirotti, V.; Giampaoli, V.; Lonardo, A. Do nonalcoholic fatty liver disease and fetuin-a play different roles in symptomatic coronary artery disease and peripheral arterial disease? Diseases 2018, 6, 17. [CrossRef] [PubMed]

3. Pan, X.; Wen, S.W.; Bestman, P.L.; Kaminga, A.C.; Acheampong, K.; Liu, A. Fetuin-A in Metabolic syndrome: A systematic review and meta-analysis. PLOS ONE 2020, 15, e0229776. [CrossRef]

4. Jensen, M.K.; Bartz, T.M.; Mukamal, K.J.; Djousse, L.; Kizer, J.R.; Tracy, R.P.; Zieman, S.J.; Rimm, E.B.; Siscovick, D.S.; Shlipak, M.; et al. Fetuin-A, type 2 diabetes, and risk of cardiovascular disease in older adults: The cardiovascular health study. Diabetes Care 2013, 36, 1222-1228. [CrossRef] [PubMed]

5. Kahraman, A.; Sowa, J.P.; Schlattjan, M.; Sydor, S.; Pronadl, M.; Wree, A.; Beilfuss, A.; Kilicarslan, A.; Altinbas, A.; Bechmann, L.P.; et al. Fetuin-A mRNA expression is elevated in NASH compared with NAFL patients. Clin. Sci. (Lond. Engl. 1979) 2013, 125, 391-400. [CrossRef] [PubMed]

6. Zhou, Z.; Sun, M.; Jin, H.; Chen, H.; Ju, H. Fetuin-a to adiponectin ratio is a sensitive indicator for evaluating metabolic syndrome in the elderly. Lipids Health Dis. 2020, 19, 61. [CrossRef]

7. Celebi, G.; Genc, H.; Gurel, H.; Sertoglu, E.; Kara, M.; Tapan, S.; Acikel, C.; Karslioglu, Y.; Ercin, C.N.; Dogru, T. The Relationship of circulating Fetuin-A with liver histology and biomarkers of systemic inflammation in nondiabetic subjects with nonalcoholic fatty liver disease. Saudi J. Gastroenterol. 2015, 21, 139-145. [CrossRef]

8. Denecke, B.; Graber, S.; Schafer, C.; Heiss, A.; Woltje, M.; Jahnen-Dechent, W. Tissue distribution and activity testing suggest a similar but not identical function of fetuin-B and fetuin-A. Biochem. J. 2003, 376, 135-145. [CrossRef]

9. Moher, D.; Liberati, A.; Tetzlaff, J.; Altman, D.G. Preferred reporting items for systematic reviews and meta-analyses: The PRISMA statement. BMJ (Clin. Res. Ed.) 2009, 339, b2535. [CrossRef]

10. Stang, A. Critical evaluation of the Newcastle-Ottawa scale for the assessment of the quality of nonrandomized studies in meta-analyses. Eur. J. Epidemiol. 2010, 25, 603-605. [CrossRef]

11. Higgins, J.P.; Thompson, S.G.; Deeks, J.J.; Altman, D.G. Measuring inconsistency in meta-analyses. BMJ (Clin. Res. Ed.) 2003, 327, 557-560. [CrossRef] [PubMed]

12. DerSimonian, R.; Laird, N. Meta-analysis in clinical trials revisited. Contemp. Clin. Trials 2015, 45, 139-145. [CrossRef] [PubMed]

13. Egger, M.; Davey Smith, G.; Schneider, M.; Minder, C. Bias in meta-analysis detected by a simple, graphical test. BMJ (Clin. Res. Ed.) 1997, 315, 629-634. [CrossRef] [PubMed]

14. Ballestri, S.; Meschiari, E.; Baldelli, E.; Musumeci, F.E.; Romagnoli, D.; Trenti, T.; Zennaro, R.G.; Lonardo, A.; Loria, P. Relationship of serum fetuin-A levels with coronary atherosclerotic burden and NAFLD in patients undergoing elective coronary angiography. Metab. Syndr. Relat. Disord. 2013, 11, 289-295. [CrossRef] [PubMed]

15. Cui, Z.; Xuan, R.; Yang, Y. Serum fetuin A level is associated with nonalcoholic fatty liver disease in Chinese population. Oncotarget 2017, 8, 107149-107156. [CrossRef] 
16. Dogru, T.; Genc, H.; Tapan, S.; Aslan, F.; Ercin, C.N.; Ors, F.; Kara, M.; Sertoglu, E.; Karslioglu, Y.; Bagci, S.; et al. Plasma fetuin-A is associated with endothelial dysfunction and subclinical atherosclerosis in subjects with nonalcoholic fatty liver disease. Clin. Endocrinol. 2013, 78, 712-717. [CrossRef]

17. Ebert, T.; Linder, N.; Schaudin, A.; Busse, H.; Berger, J.; Lichtinghagen, R.; Keim, V.; Wiegand, J.; Karlas, T. Association of fetuin B with markers of liver fibrosis in nonalcoholic fatty liver disease. Endocrine 2017, 58, 246-252. [CrossRef]

18. Şiraz, Ü.G.; Doğan, M.; Hatipoğlu, N.; Muhtaroğlu, S.; Kurtoğlu, S. Can fetuin-A be a marker for insulin resistance and poor glycemic control in children with type 1 diabetes mellitus? JCRPE J. Clin. Res. Pediatr. Endocrinol. 2017, 9, 293-299. [CrossRef]

19. Haukeland, J.W.; Konopski, Z.; Aasheim, E.; Johansen, O.E.; Løberg, E.M.; Haaland, T.; Gladhaug, I.; Dahl, T.; Yndestad, A.; Aukrust, P.; et al. Elevated levels of fetuin a in nonalcoholic fatty liver disease. J. Hepatol. 2010, 52, S143-S144. [CrossRef]

20. Haukeland, J.W.; Dahl, T.B.; Yndestad, A.; Gladhaug, I.P.; Løberg, E.M.; Haaland, T.; Konopski, Z.; Wium, C.; Aasheim, E.T.; Johansen, O.E.; et al. Fetuin A in nonalcoholic fatty liver disease: In vivo and in vitro studies. Eur. J. Endocrinol. 2012, 166, 503-510. [CrossRef]

21. El-Ashmawy, H.M.; Ahmed, A.M. Serum fetuin-B level is an independent marker for nonalcoholic fatty liver disease in patients with type 2 diabetes. Eur. J. Gastroenterol. Hepatol. 2019, 31, 859-864. [CrossRef]

22. Kurtoglu, S.; Dogan, M.; Hatipoglu, N.; Muhtaroglu, S.; Doganay, S.; Gul, U.; Elmali, F. Serum fetuin-a level for diagnosis hepatic steatosis in children with type 1 diabetes mellitus. Horm. Res. Paediatr. 2015, 84, 281-282.

23. Lebensztejn, D.M.; Białokoz-Kalinowska, I.; Kłusek-Oksiuta, M.; Tarasów, E.; Wojtkowska, M.; Kaczmarski, M. Serum fetuin A concentration is elevated in children with non-alcoholic fatty liver disease. Adv. Med. Sci. 2014, 59, 81-84. [CrossRef]

24. von Loeffelholz, C.; Horn, P.; Birkenfeld, A.L.; Claus, R.A.; Metzing, B.U.; Döcke, S.; Jahreis, G.; Heller, R.; Hoppe, S.; Stockmann, M.; et al. Fetuin A is a predictor of liver fat in preoperative patients with nonalcoholic fatty liver disease. J. Investig. Surg. 2016, 29, 266-274. [CrossRef]

25. Li, Z.; Lin, M.; Liu, C.; Wang, D.; Shi, X.; Chen, Z.; Liu, Y.; Yang, S.; Li, X. Fetuin-B links nonalcoholic fatty liver disease to type 2 diabetes via inducing insulin resistance: Association and path analyses. Cytokine 2018, 108, 145-150. [CrossRef]

26. Lin, M.; Liu, C.; Liu, Y.; Wang, D.; Shi, X.; Chen, Z.; Liu, J.; Zheng, C.; Li, X.; Yang, S.; et al. Fetuin-B links nonalcoholic fatty liver disease to chronic kidney disease in obese Chinese adults: A cross-sectional study. Diabetes Metab. Res. Rev. 2018, 34, 50. [CrossRef]

27. Mondal, S.A.; Dutta, D.; Kumar, M.; Singh, P.; Basu, M.; Selvan, C.; Mukhopadhyay, S. Neck circumference to height ratio is a reliable predictor of liver stiffness and nonalcoholic fatty liver disease in prediabetes. Indian J. Endocrinol. Metab. 2018, 22, 347-354.

28. Nascimbeni, F.; Romagnoli, D.; Baldelli, E.; Sirotti, V.; Ballestri, S.; Bertolotti, M.; Lonardo, A. Nafld, Fetuin-A and Atherosclerosis Burden: Does Vascular Topography Matter? J. Hepatol. 2015, 62, S748-S749. [CrossRef]

29. Ou, H.Y.; Yang, Y.C.; Wu, H.T.; Wu, J.S.; Lu, F.H.; Chang, C.J. Increased fetuin-A concentrations in impaired glucose tolerance with or without nonalcoholic fatty liver disease, but not impaired fasting glucose. J. Clin. Endocrinol. Metab. 2012, 97, 4717-4723. [CrossRef]

30. Ou, H.Y.; Wu, H.T.; Hung, H.C.; Yang, Y.C.; Wu, J.S.; Chang, C.J. Endoplasmic reticulum stress induces the expression of fetuin-A to develop insulin resistance. Endocrinology 2012, 153, 2974-2984. [CrossRef]

31. Pampanini, V.; Inzaghi, E.; Germani, D.; Alterio, A.; Puglianiello, A.; Alisi, A.; Nobili, V.; Cianfarani, S. Serum Fetuin-A levels in obese children with biopsy proven nonalcoholic fatty liver disease. Nutr. Metab. Cardiovasc. Dis. 2018, 28, 71-76. [CrossRef] [PubMed]

32. Rametta, R.; Ruscica, M.; Dongiovanni, P.; Macchi, C.; Fracanzani, A.L.; Steffani, L.; Fargion, S.; Magni, P.; Valenti, L. Hepatic steatosis and PNPLA3 I148M variant are associated with serum Fetuin-A independently of insulin resistance. Eur. J. Clin. Investig. 2014, 44, 627-633. [CrossRef] [PubMed]

33. Reinehr, T.; Roth, C.L. Fetuin-A and its relation to metabolic syndrome and fatty liver disease in obese children before and after weight loss. J. Clin. Endocrinol. Metab. 2008, 93, 4479-4485. [CrossRef]

34. Roshdy, A.; Okash, H.; Soliman, A.; Maamoun, H.; Shaker, O.; Soliman, M.A.; Hamdy, A. Serum Fetuin A levels: Are They a reliable marker for hepatic steatosis and regional adiposity in renal transplant recipients? Transplant. Proc. 2015, 47, 2703-2706. [CrossRef] 
35. Sato, M.; Kamada, Y.; Takeda, Y.; Kida, S.; Ohara, Y.; Fujii, H.; Akita, M.; Mizutani, K.; Yoshida, Y.; Yamada, M.; et al. Fetuin-A negatively correlates with liver and vascular fibrosis in nonalcoholic fatty liver disease subjects. Liver Int. Off. J. Int. Assoc. Study Liver 2015, 35, 925-935. [CrossRef]

36. Sonmez, A.; Nikolic, D.; Dogru, T.; Ercin, C.N.; Genc, H.; Cesur, M.; Tapan, S.; Karslioglu, Y.; Montalto, G.; Banach, M.; et al. Low- and high-density lipoprotein subclasses in subjects with nonalcoholic fatty liver disease. J. Clin. Lipidol. 2015, 9, 576-582. [CrossRef]

37. Tanoglu, A.; Akyol, T.; Karagoz, E.; Yazgan, Y. The relationship between serum fetuin a and liver fibrosis in patients with non alcoholic fatty liver disease (NAFLD). Hepatol. Int. 2015, 9, S359.

38. Wong, V.W.S.; Wong, G.L.H.; Chan, H.Y.; Yeung, D.K.W.; Chan, R.S.M.; Chim, A.M.L.; Chan, C.K.M.; Tse, Y.K.; Woo, J.; Chu, W.C.W.; et al. Bacterial endotoxin and non-alcoholic fatty liver disease in the general population: A prospective cohort study. Aliment. Pharmacol. Therap. 2015, 42, 731-740. [CrossRef]

39. Yilmaz, Y.; Yonal, O.; Kurt, R.; Ari, F.; Oral, A.Y.; Celikel, C.A.; Korkmaz, S.; Ulukaya, E.; Ozdogan, O.; Imeryuz, N.; et al. Serum fetuin A/ $\alpha 2 \mathrm{HS}$-glycoprotein levels in patients with non-alcoholic fatty liver disease: Relation with liver fibrosis. Ann. Clin. Biochem. 2010, 47, 549-553. [CrossRef]

40. Zhu, J.; Wan, X.; Wang, Y.; Zhu, K.; Li, C.; Yu, C.; Li, Y. Serum fetuin B level increased in subjects of nonalcoholic fatty liver disease: A case-control study. Endocrine 2017, 56, 208-211. [CrossRef]

41. Kim, M.; Lim, S.J.; Lee, H.-J.; Kim, S.Y.; Nho, C.W. Gomisin J Inhibits oleic acid-induced hepatic lipogenesis by activation of the AMPK-dependent pathway and inhibition of the hepatokine Fetuin-A in HepG2 cells. J. Agric. Food Chem. 2015, 63, 9729-9739. [CrossRef] [PubMed]

42. Trepanowski, J.F.; Mey, J.; Varady, K.A. Fetuin-A: A novel link between obesity and related complications. Int. J. Obes. 2015, 39, 734-741. [CrossRef]

43. Jung, T.W.; Youn, B.S.; Choi, H.Y.; Lee, S.Y.; Hong, H.C.; Yang, S.J.; Yoo, H.J.; Kim, B.H.; Baik, S.H.; Choi, K.M. Salsalate and adiponectin ameliorate hepatic steatosis by inhibition of the hepatokine fetuin-A. Biochem. Pharmacol. 2013, 86, 960-969. [CrossRef]

44. Robinson, K.N.; Vazquez-Vidal, I.; Marques, C.; Andrade, F.C.D.; Aradillas-Garcia, C.; Teran-Garcia, M. Circulating triglycerides and the association of triglycerides with dietary intake are altered by alpha-2-heremans-schmid glycoprotein polymorphisms. J. Nutrigenet. Nutrigenom. 2017, 10, 75-83. [CrossRef]

45. Kahraman, A.; Schlattjan, M.; Pronadl, M.; Wree, A.; Sydor, S.; Sowa, J.P.; Bechmann, L.; Syn, W.K.; Gerken, G.; Canbay, A. Reduction of fetuin-A in morbidly obese patients with NAFLD following weight loss by bariatric surgery. Hepatology 2012, 56, 882A.

46. Alam, S.; Anam, K.; Islam, S.; Mustafa, G.; Mamun, A.A.; Ahmad, N. Clinical, anthropometric, biochemical and histological character of nonalcoholic fatty liver disease without Insulin Resistance. J. Clin. Exp. Hepatol. 2019, 9, 176-181. [CrossRef] [PubMed]

47. Verras, C.G.; Christou, G.A.; Simos, Y.V.; Ayiomamitis, G.D.; Melidonis, A.J.; Kiortsis, D.N. Serum fetuin-A levels are associated with serum triglycerides before and 6 months after bariatric surgery. Hormones (Athens Greece) 2017, 16, 297-305. [PubMed]

48. Marhaug, G.; Shah, V.; Shroff, R.; Varsani, H.; Wedderburn, L.R.; Pilkington, C.A.; Brogan, P.A. Age-dependent inhibition of ectopic calcification: A possible role for fetuin-A and osteopontin in patients with juvenile dermatomyositis with calcinosis. Rheumatology (Oxf. Engl.) 2008, 47, 1031-1037. [CrossRef] [PubMed]

49. Chattopadhyay, M.; Mukherjee, S.; Chatterjee, S.K.; Chattopadhyay, D.; Das, S.; Majumdar, S.S.; Mukhopadhyay, S.; Mukherjee, S.; Bhattarcharya, S. Impairment of energy sensors, SIRT1 and AMPK, in lipid induced inflamed adipocyte is regulated by Fetuin A. Cell. Signal. 2018, 42, 67-76. [CrossRef] [PubMed]

50. Ampuero, J.; Ranchal, I.; Gallego-Duran, R.; Pareja, M.J.; Del Campo, J.A.; Pastor-Ramirez, H.; Rico, M.C.; Picon, R.; Pastor, L.; Garcia-Monzon, C.; et al. Oxidized low-density lipoprotein antibodies/high-density lipoprotein cholesterol ratio is linked to advanced non-alcoholic fatty liver disease lean patients. J. Gastroenterol. Hepatol. 2016, 31, 1611-1618. [CrossRef]

(C) 2020 by the authors. Licensee MDPI, Basel, Switzerland. This article is an open access article distributed under the terms and conditions of the Creative Commons Attribution (CC BY) license (http://creativecommons.org/licenses/by/4.0/). 